\title{
Análisis de la apropiación y uso de las TIC por parte de las pymes colombianas*
}

\author{
J. Ricardo Puentes $G^{* *}$
}

Recibido: 7 de septiembre de 2016 • Aprobado: 5 de octubre de 2016

\section{Resumen}

El desarrollo y la apropiación de las TIC (tecnologías de la información y de las comunicaciones) han marcado el rumbo de las naciones en la medida en que estas han asumido dichas tecnologías en todos los niveles. Los innumerables beneficios y promesas de la apropiación de estas tecnologías por parte de los ciudadanos, ante todo de las empresas que componen una economía, al parecer aún se hacen esperar.

Colombia no ha sido indiferente al proceso del desarrollo de las TIC. Mediante la creación de políticas y leyes enfocadas a buscar la masificación del uso de tecnologías, orientadas tanto al usuario final como al sector productivo, el país trata de incrustarse en exigentes procesos para tratar de asumirlas. Las diversas tecnologías computacionales y de información conforman el eje central del desarrollo comercial de las empresas; pero, para el caso colombiano, los esfuerzos llevados a cabo por los últimos gobiernos no han dado los resultados esperados, sobre todo en lo que concierne a las pequeñas y medianas empresas (pymes), como lo confirma la presente investigación.

Palabras clave: Pymes, TIC, tecnologías, información, comunicaciones, desarrollo, apropiación, usos.

"Artículo producto del proyecto de investigación "Apropiación y uso de las TIC por parte de las pymes colombianas", llevado a cabo dentro del grupo GESEF (Grupo de Estudios Socio-Económicos y Financieros), de la Facultad de Finanzas y Comercio Internacional de la Corporación Universitaria Republicana. DOI: http://dx.doi.org/10.15332/s1900-0448.2017.0046.01

"Administrador público, y magíster en Dirección Estratégica. Docente investigador en la Corporación Universitaria Republicana y docente catedrático en el Departamento de Administración de Empresas de la Universidad Central. Correos electrónicos: rpuentesg@urepublicana.edu.co,rpuentesg@ ucentral.edu.co. 


\title{
Analysis of the adoption and use of ICT by Colombian SMEs
}

\begin{abstract}
The development and appropriation of ICT (Information and Communication Technologies) has marked the course of the nations as they take on these technologies at all levels. The innumerable benefits and promises of the appropriation of these technologies by the citizens and, above all, of the companies that make up an economy seem to be still waiting.

Colombia has not been indifferent to the process of ICT development, through the creation of policies and laws aimed at seeking the massification of the use of technologies, aimed at both the end user and the productive sector, the country tries to embed itself in demanding processes to deal with of assuming them. The diverse computational and information technologies, are the central axis of the commercial development of the companies; But for the Colombian case the efforts made by the last governments have not yielded the expected results, especially in the case of small and medium-sized enterprises (SMEs), as confirmed by the present investigation.
\end{abstract}

Keywords: SMEs, ICT, technologies, information, communications, development, appropriation, uses.

\section{Análise da Apropriação e uso das TIC por parte das PME COLOMBIANAS}

\section{J Ricardo Puentes G.}

\section{Resumo}

O desenvolvimento e apropriação das TIC (Tecnologias da Informação e da Comunicação) tem marcado o rumo das nações na medida em que estas têm assumido estas tecnologias em todos os níveis. Os inúmeros benefícios e promessas da apropriação de estas tecnologias por parte dos cidadãos, ante todo das empresas que compõem uma economia ao parecer ainda se fazem esperar.

Colômbia não tem sido indiferente ao processo do desenvolvimento das TIC. Mediante a criação de políticas e leis focadas em buscar a massificação do uso de 
tecnologias, orientadas tanto ao usuário final como ao setor produtivo, o país tenta se inserir em exigentes processos para tratar de assumi-las. As diversas tecnologias computacionais e de informação conformam o eixo central do desenvolvimento comercial das empresas; mas, para o caso colombiano, os esforços feitos pelos últimos governos não tem oferecido os resultados esperados, sobre todo no relacionado com as pequenas e médias empresas (PME) como o confirma a seguinte pesquisa.

Palavras-chave: PME, TIC, tecnologias, informação, comunicação, desenvolvimento, apropriação, usos.

\section{Introducción}

El presente análisis sobre la apropiación y el uso de las TIC por parte de las pymes colombianas es producto de los resultados de la investigación en torno a las pymes y el uso que estas hacen de las TIC (tecnologías de la información y de las comunicaciones) en Colombia. La investigación, de carácter descriptivo, parte de la encuesta realizada por el DANE en el año 2001, por medio de la cual se realizó un censo y un exhaustivo diagnóstico de las pymes en términos del uso y la apropiación de las TIC; los resultados obtenidos por el DANE se relacionaron con las actuales encuestas y estudios, con el fin de observar la real apropiación de estas tecnologías a través de estos años. estudiarla investigación pretende abordar el uso que están haciendo las pymes de las TIC y finalmente determinar las causas por las cuales se estén o no apropiando las TIC.

Al hacer un análisis sobre los resultados de la encuesta realizada por el DANE en 2001 \ la cual, hay que reafirmarlo fue bastante amplia $\bigotimes$, se refleja el atraso que por ese entonces estaba experimentando el parque empresarial de las pequeñas, medianas y micro empresas colombianas. Se observa que esta tendencia no ha sido superada con el correr de los años, a pesar de los esfuerzos y de las diferentes iniciativas de los últimos gobiernos, como quedó confirmado al comparar los actuales estudios y encuestas con el trabajo del DANE en 2001.

La investigación además se enmarca dentro de la perspectiva teórica de diversos autores y sus respectivos enfoques, que, en general, van desde el marcado optimismo de los que ven en el desarrollo de los actuales sistemas computacionales, tecnológicos y de información la panacea para los males de la humanidad y, en consecuencia, 
de los países en desarrollo, hasta los más pesimistas, que ven un caos que engloba todo un sistema totalizante, monopolístico y dependiente (Negroponte, 1995; Dertouzos, 1998). En concordancia con lo dispuesto anteriormente, el profesor Acosta, ha dispuesto:

Las nuevas realidades políticas y económicas ameritan repensar las categorías básicas del derecho de contratos, especialmente sus principios generales. A tal punto, que es imprescindible reconocer que los principios y las tendencias que dominan la teoría posmoderna del contrato en algunos ámbitos se complementan, pero de manera indudable, en otros aspectos se contradicen ampliamente. (Acosta, 2012, p.1).

\section{Reflexión}

Coincidimos con el criterio de Flores (2014) en el sentido de que

No hay duda de que la conectividad y el acceso a nuevas tecnologías de información han crecido vertiginosamente en Colombia durante los últimos años y que, aunque aún nos falta bastante para estar al nivel de países líderes, como los europeos, es indudable que vamos por un buen camino. (Flores, 2014, p. 44).

De conformidad con Velásquez y Moreno (2015):

Una de las garantías del Estado social de derecho y democrático condensado en la sociedad de la información es que los beneficios de las tecnologías de la información y las comunicaciones (TIC) sean accesibles a todos los ciudadanos, en condiciones de igualdad. (Velásquez y Moreno, 2015, p.139).

En este sentido, con más de 6 millones de desplazados y un índice de desigualdad del 0,5 (según el coeficiente de Gini en en que 0 es la perfecta igualdad y 1 es desigualdad total) que deja al país en el tercer lugar en la región después de Haití y Brasil, sin contar con los recurrentes problemas de narcotráfico, distribución de tierras y la pobreza reinante, Colombia le apuesta su desarrollo al avance de las TIC y se centra de esta forma en el marco y la filosofía de los países que ven con 
optimismo la salida de sus males alrededor de la implementación de las diferentes tecnologías desarrolladas alrededor de las TIC.

El impacto producido por el fenómeno de la globalización y la internacionalización de las economías se ha sentido en todos los países del mundo y los ha forzado a adoptar nuevos mecanismos que les permitan prepararse para ser más productivos y competitivos.

En consecuencia, los países adoptan nuevas legislaciones y mecanismos en todos los frentes, que van desde la reforma a los sistemas educativos hasta grandes obras de infraestructuras viales, portuarias, tecnológicas y de comunicaciones, entre otras, que les permitan asumir los nuevos sistemas productivos y de relaciones. Pero es tal vez el desarrollo de las tecnologías que giran alrededor de la información y las comunicaciones el que impele a las sociedades y organizaciones, económicas o no, a transformarse y adaptarse con mayor rapidez a los nuevos cambios.

Ahora bien, el desarrollo de la tecnología ha logrado afectar profundamente el comportamiento de las personas, quienes actualmente cuentan con una amplia gama de sistemas de comunicación e interrelaciones, rompiendo el aislamiento y formando comunidades cada día más informadas y, por lo tanto, más exigentes. En el caso de las organizaciones, el desarrollo de nuevas tecnologías ha permitido que estas se hagan cada vez más eficientes, respondan con mayor dinamismo a los cambios, que minimicen sus costos de producción y, sobre todo, que den respuestas acertadas y satisfactorias a sus clientes que, en suma, son los que buscan optimizar su inversión en la adquisición de un servicio y/o producto. Además, está la ampliación de los mercados, antes de carácter local y ahora extendidos con los procesos de globalización por medio de los diferentes acuerdos o tratados de libre comercio entre países.

Colombia no ha sido indiferente a este proceso. Mediante la creación de políticas y leyes enfocadas en buscar la masificación del uso de tecnologías, orientadas tanto al usuario final como al sector productivo, el país trata de incrustarse en los exigentes procesos para tratar de asumirlas. Las diversas tecnologías computacionales y de información conforman el eje del desarrollo comercial, logístico y productivo de las actuales empresas en un mundo cada vez más interconectado.

La pregunta es si todos estos esfuerzos se ven reflejados en un mejoramiento de la vida de los colombianos o si estos responden a las reales necesidades del país y a sus expectativas. Por otra parte, en lo que concierne a la inclusión y al uso de 
las empresas, en particular las mypimes en lo referente a las TIC, los resultados no parecen ser muy alentadores a pesar de los esfuerzos de los últimos gobiernos.

A partir de la encuesta realizada por el DANE en convenio con la Agencia de Conectividad de la época en el año 2001 y los actuales informes realizados por empresas desarrolladoras de tecnologías computacionales y de las comunicaciones, gremios y el mismos DANE, se hace un análisis de la evolución y actual situación de las mipymes nacionales. La investigación propuesta, como se expresó en la introducción de la presente reflexión, se enmarca dentro de la perspectiva teórica de diversos autores y sus respectivos enfoques, que, en general, van desde un marcado optimismo de los que ven en el desarrollo de los actuales sistemas computacionales, tecnológicos y de información la panacea para los males de la humanidad y, en consecuencia, de los países en desarrollo, hasta los más pesimistas, que ven un caos que engloba todo un sistema totalizante, monopolístico y dependiente (Negroponte, 1995, Dertouzos, 1998).

$\mathrm{Al}$ frente de estos cuestionamientos se encuentra Internet, la red que ha permitido el desarrollo, tal vez desaforado, de los sistemas computacionales y de información, pero también el medio ideal en el que se concentra la mayoría de estas tecnologías y del que se han derivado múltiples usos y oportunidades.

Los más optimistas muestran la red como un espacio libre, de comunicación, democrático y con una gran capacidad para transformar e incrementar la producción en la economía, que podría ser empleada para incrementar la participación de las minorías en las decisiones nacionales, o bien, para hacerse escuchar en los casos de situaciones injustas o de discriminación (Negroponte, 1995). También puede ser útil como un sistema ideal en la dirección transparente de la administración pública y, ante todo, como el medio por el cual las pequeñas empresas pueden salir a competir en iguales condiciones frente a las grandes empresas. En otras palabras, la red sería el medio que permitiría cerrar la brecha en términos de participación democrática y de la distribución de la riqueza en el mundo. El mejor ejemplo para apoyar la postura optimista es ver el desarrollo y las consecuencias de la llamada Primavera Árabe. En concordancia con lo dispuesto anteriormente, Palomares ha considerado que:

Si bien el comercio electrónico no es un contrato en sí mismo, sino un factor esencial para la existencia e interacción de la nueva Lex Mercatoria en el contexto global, 
este se ha constituido en el segundo escenario de realización de contratos. (Palomares, 2012, p. 1).

En el otro extremo está el enfoque pesimista, compuesto por quienes ven el peligro de que Internet podría caer bajo el control de las grandes empresas, del poder político o, peor aún, en manos de individuos, en cuyo caso se podría convertir en un instrumento de control social que terminaría ampliando aún más la brecha existente entre ricos y pobres. Nicolás Negroponte, director del Laboratorio de Medios del Instituto Tecnológico de Massachussets y Hogar de la Web, afirma:

El único peligro que acecha a la Net es el gobierno y los políticos que quieren controlarla. Por lo general, bajo el pretexto de querer convertir a la Net en un lugar seguro para los niños, hay gente, en todo el mundo, que intenta permanentemente censurar sus contenidos. Y peor aún, algunos países -incluyendo a los Estados Unidos- quieren asegurarse de que exista alguna posibilidad de escuchar los mensajes, es decir, que se les permita interferir los mensajes, como si fueran comunicaciones telefónicas. (Negroponte, 1995, p. 6).

Este comentario del texto arriba citado corresponde a la edición de 1995. Luego de casi veinte años se confirma su predicción sobre todo lo referente al polémico proyecto de ley antipiratería promovido por el Congreso Norteamericano, conocido como "SOPA" (Stop Online Piracy Act), es decir, acta para detener la piratería en Internet, el cual finalmente no pasó debido a las protestas que generó por parte de la población y diversas entidades prestadoras de servicios en la red. Mientras que, por un lado, el Gobierno norteamericano trataba de legalizar el espionaje, por el otro ya se concretaban sus intenciones o la concreción de estas afirmaciones con la actual polémica por la interceptación masiva de datos denunciada por el excontratista de seguridad Edward Snowden, que ya hace realidad las preocupaciones expresadas por Negroponte o más “antiguas”, como las expuestas por Orson Wells en su libro 1984. Colombia tampoco ha sido ajena a esta problemática, hay que recordar la polémica Ley Lleras, que tuvo que ser archivada por las controversias que generó en términos de derechos de autor y propiedad intelectual.

Para una mejor ilustración sobre las actuales consideraciones y preocupaciones en torno al desarrollo y a la aplicación de las tecnologías de información y de las comunicaciones (TIC), veamos un aparte del informe del Center for Internacional 
Development (CID) de la Universidad de Harvard, que se encuentra en Preparación para un mundo interconectado. Una guía para los países en desarrollo:

Los logros tecnológicos de las últimas décadas son la razón principal de la asombrosa nueva oleada de riqueza y bienestar en los países más ricos del mundo. El comercio electrónico y las aplicaciones de tecnologías de información y comunicación (TIC) se han convertido en poderosos motores de crecimiento económico e incrementos de productividad y están transformando la estructura del mundo en que vivimos. Sin embargo, el mundo desarrollado está cosechando la gran mayoría de estas ganancias. Según nuestro punto de vista, el uso apropiado de las tecnologías de la información y las comunicaciones puede mejorar la vida del $80 \%$ de la población mundial que vive en los países en desarrollo.

La gran división entre países ricos y pobres, ampliamente conocida en lo que respecta a aspectos sociales y de riqueza, prevalece y es igualmente preocupante en el ámbito de las tecnologías de la información y las comunicaciones. Mientras el crecimiento del Internet y la continua "digitalización de la sociedad" son eventos destacados en países más desarrollados, muchos de los líderes de las naciones en desarrollo quedan preguntándose cómo participar de los rápidos cambios que están sucediendo. ¿Cómo pueden las TIC ayudar a los gobiernos y a las comunidades a volverse más productivos? ¿Cómo prepararse para participar del mundo interconectado?

Creemos que hoy existe una oportunidad única para que muchas de estas comunidades se vinculen a las redes globales de información y obtener el impulso necesario hacia mayor riqueza y prosperidad. Sin embargo, sin un esfuerzo conjunto del mundo en desarrollo de preparación para la nueva economía, las brechas entre los niveles de vida de los países desarrollados y en vía de desarrollo se ampliará y el uso de estas tecnologías se mantendrá como un fenómeno limitado, en su mayor parte, a las comunidades más ricas del mundo.

En cualquiera de los casos, una cosa es bien cierta: para que la red sirva a los ciudadanos y a las empresas en igualdad de condiciones, estos tienen que tener los medios que les permitan acceder a ella, esto es, los sistemas de cómputo, los proveedores de acceso y, adicionalmente, la capacitación necesaria para el uso de la tecnología, junto con el software requerido para el máximo aprovechamiento.

Aquí es donde es importante el papel que cumplen los gobiernos de los diferentes países en la medida en que generen la infraestructura necesaria para masificar el uso 
del Internet y de los sistemas computacionales y de información, lo cual, al parecer, no es consistente con la realidad, y más bien prevalece la visión pesimista, como se puede observar en las estadísticas globales en torno a la cobertura de la Internet, en las que se evidencia una brecha cada vez más amplia entre los países desarrollados y los que no lo son (para una mayor comprensión, ver el reporte de http://www. internetworldstats.com/stats.htm, el cual muestra estadísticas consistentes con el grado de desarrollo de los países).

Una de las características más relevantes del uso de la Internet es el acceso a la información (Dertouzos, 1998), pero el valor de esta información depende de las necesidades del individuo. En las naciones desarrolladas, en donde los ciudadanos cuentan con unos ingresos altos que les dan la posibilidad de adquirir una amplia gama de servicios y productos con toda comodidad, el valor que le otorgarán a la información será alto, sus necesidades básicas ya están cubiertas en forma casi cotidiana y esto les permite ascender a una escala más sofisticada de necesidades (Maslow); pero en los países en vías de desarrollo o para los más pobres, los individuos luchan a diario para suplir sus necesidades básicas, así que ellos le otorgarán a la información un valor más bajo y, en algunos casos, seguramente ni la contemplarán: "la penosa conclusión es que, abandonado a sus propios mecanismos, el mercado de la información incrementará el abismo entre países ricos y pobres, así como entre la gente rica y pobre" (Dertouzos, 1998).

\section{Radiografía de las pymes colombianas}

Ahora bien, independientemente del enfoque y de su cobertura poblacional, las cifras son las que muestran la realidad y, para el presente caso, materia de reflexión, lo que más preocupa del tema es ver cómo las mipymes en Colombia siguen muy atrasadas en la apropiación de estas tecnologías, tal como lo confirman las diferentes encuestas realizadas por el DANE, los estudios de la Cámara de Comercio de Bogotá, encuestas de empresas especializadas en desarrollo de tecnologías y los estudios internacionales.

Las pymes son consideradas la piedra angular de toda economía, sobre ellas se soporta la mayor generación de empleo y juegan un papel importante en la producción industrial y de bienes y servicios. Si bien es cierto que las grandes industrias tienen un gran peso en el producto interno bruto de los países, estas dependen también en buena parte del soporte de las pymes. 
Para los países en desarrollo, las pymes se encuentran en constante amenaza ante el avance desaforado de los procesos de globalización. Las economías frágiles de estos países tienen que avocarse a apalancar con todos los medios a estas empresas. En Colombia, en particular, se ha desarrollado toda una legislación que pretende apalancar las pymes en pos de su crecimiento, como el Sistema Nacional de Mipymes, presentado en el artículo 3. ${ }^{\circ}$ de la Ley 905 de 2004, modificatorio del artículo 3. ${ }^{\circ}$ de la Ley 590 de 2000, el cual es un instrumento de apoyo a la micro, pequeña y mediana empresa, y está conformado por:

1. Los Consejos Regionales, reglamentados mediante Resolución 3205 de 2008 del Ministerio de Comercio Industria y Turismo.

2. El Consejo Superior de Microempresa, reglamentado mediante el Acuerdo 01 de 2012 y el Acuerdo 02 de 2013, expedido por el Presidente de dicho Consejo.

3. E1 Consejo Superior de Pequeña y Mediana Empresa, cuyos estatutos internos fueron modificados por medio del Acuerdo 01 de 2012 y el Acuerdo 02 de 2013, expedido por su presidente.

4. Los Consejos Superiores de Microempresa y de Pyme, en cumplimiento de la Norma Andina (Resolución 1487 de 2012 "Reglamento de Funcionamiento del Observatorio Andino de la MIPYME (OBAPYME)”), expidieron el Acuerdo 01 de 2013, "por el cual el Consejo Superior de Microempresa y el Consejo Superior de Pequeña y Mediana Empresa asumen las funciones del Comité Nacional Interinstitucional OBAPYME”.

A la par de los avances económicos, en términos de lo que dicta la globalización van los desarrollos tecnológicos, técnicos y científicos, entre ellos, las tecnologías de la información y de las comunicaciones (TIC), derivadas a la par del desaforado desarrollo de la Internet y que han permitido generar nuevas relaciones en todos los ámbitos sociales, gubernamentales y económicos e impelen a las empresas a sumirlas so pena de quedar rezagadas en sus procesos administrativos, productivos y de mercadeo o, finalmente, condenadas a salir en forma definitiva del mercado.

La nueva sociedad de la información - parodiando a Drucker-genera relaciones muy complejas y demandas de diversa índole en las sociedades. Las pymes, en su quehacer cotidiano, deben responder a estas demandas. Deben responder a la 
sociedad con la demanda incesante de bienes y servicios. Las TIC permiten hacer más eficientes y menos costosos los procesos productivos y responder con eficiencia a las demandas de los consumidores: cada día son más los individuos que se atreven a hacer compras a través de la red; crecen las empresas que hacen sus pedidos de materias primas y, en general, para cubrir muchas de sus necesidades de tipo administrativo usando la Internet; el desarrollo de los procesos administrativos e impositivos por parte de los gobiernos, conocido como el e-gobierno, obliga a que las empresas tengan que hacer uso de ella, pues se ven obligadas a relacionarse con su entorno y a hacer uso de este canal. Para el caso de las pymes, este puede ser un instrumento ideal para su sostenimiento y sobrevivencia o, por el contrario, de no asumirlas, para su desaparición del mercado. Es por esto que la relación pymes y TIC es relevante e inevitable. A nivel nacional, la diferencia entre asumirlas o no es simplemente la ampliación de la brecha entre las economías desarrolladas y las que no lo son.

Algunas de las características más importantes de las mipymes colombianas son:

- Altos niveles de informalidad

- Bajos niveles de asociatividad

- Estrechez de los mercados a los que dirigen sus productos

- Bajo nivel tecnológico y de formación de sus recursos humanos

- Limitado acceso al sector financiero

- Cautelosos para invertir

- Baja capacidad de innovación

- Limitada participación en el mercado de la contratación pública

- Bajo uso de tecnologías de la información y las comunicaciones (TIC). (Resaltado personal) (MINTIC, 2011).

\section{Las pymes y las TIC en cifras}

Según el Reporte Global de Tecnologias de la Información (The Global Information Technology Report) del Foro Económico Mundial, que desarrolló un instrumento que permite determinar la debilidad o fortaleza de un país en el potencial uso de las TIC por medio de la medición del grado de preparación de los países para participar y beneficiarse de los desarrollos de las TIC, instrumento denominado Índice de Potencial para la Conectividad (Networked Readiness Indes - NRI) 
(DNP, 2012a), en el último informe se evaluaron 143 países, y los diez primeros en el índice de disponibilidad para conectividad (NRI) (DNP, 2012a) son solo países desarrollados; mientras tanto, "América Latina y el Caribe siguen sufriendo un notable retraso en la adopción de las TIC. En el ranking global, ningún país de la región llega a la posición de los 30 mejores del mundo" (DNP, 2012a). Del mismo informe se destaca que tan solo dos países latinoamericanos están entre los primeros 50 lugares, estos son Chile y Uruguay.

Para el caso concreto de Colombia, en el Reporte Global de Tecnologías de la Información (RGTI) de 2015 que realiza anualmente el Foro Económico Mundial, nuestro país ocupó el puesto 64 entre 143 economías. El reporte destaca el avance que el país ha logrado alrededor de tres aspectos: entornos de las TIC, disponibilidad de las TIC y uso de las TIC. De esta forma, Colombia presenta un sensible avance con respecto a años anteriores, dado que en 2012 se ubicó en el puesto 73 de los 142 países analizados, pues en la evaluación de la época se afirma: "el país todavía sufre de serios problemas que dificultan su capacidad para aprovechar las TIC. Existe un significativo rezago en infraestructura y acceso a contenidos digitales como texto, audiovisual y software" (DNP, 2012a). Hay que destacar que la anterior afirmación sobre el rezago en el aprovechamiento de las TIC no fue solo sobre Colombia, sino acerca de la mayoría de los países de América Latina en 2012. En el reporte de ese mismo año también se afirma: "junto con unas condiciones poco favorables para el emprendimiento y la innovación (95), lo cual se refleja en una baja utilización de las TIC por las empresas (71)" (DNP, 2012a). Como se puede observar, el panorama no es muy alentador, máxime con la afirmación del actual Ministro de las Comunicaciones, Diego Molano Vega: "De los 1.6 millones de empresas que hay en el país, 97 por ciento son micro, pequeñas y medianas. Apenas un 7 por ciento de ellas están conectadas a Internet" (Portafolio, 24 de mayo de 2012). Pero, a paso lento, al parecer las políticas gubernamentales de los últimos años están dando algunos frutos.

El bajo uso de herramientas TIC básicas por parte de las microempresas se explica principalmente por la percepción de que tales instrumentos no resultan útiles para su negocio; más del 80.0 \% esgrime esta razón (CCIT y Fedesarrollo, 2013).

La más reciente encuesta sobre los microestablecimientos (que son la mayoría del parque empresarial colombiano), realizada por el DANE, muestra un penoso avance de estas entidades en el uso de las TIC para el primer trimestre de 2016: en general, un $32.4 \%$ de los microestablecimientos está haciendo uso de algún 
bien TIC; las dedicadas al comercio, en un $24.3 \%$; las de industria, el $39 \%$ y las dedicadas a los servicios son las que presentan un mayor uso de TIC, con un 46,4 $\%$. Como se puede observar, estamos aún lejos de que por lo menos la mitad de las microempresas asuma las TIC.

Solo estos datos dejan un esbozo de la distancia que aún hay por parte de las mipymes para asumir las TIC como un componente de su desarrollo. Otra reflexión al margen: supongamos que un buen número de pymes deciden adoptar con todas las reglas las TIC, empezando por el desarrollo de un sitio en la red, con las seguridades correspondientes para hacer ventas, los vínculos con los sistemas de pagos y bancarios, la presentación del sitio en dos idiomas, etc. Las posibilidades de hacer negocios son múltiples, dado el efecto que tiene un sitio en la red. Ahora bien: ¿cuáles son las posibilidades de respuesta que tienen las pymes y las mipymes nacionales para el mercado mundial?

Una mirada al uso de las tecnologías de la información y las comunicaciones en Colombia nos ofrece una visión más clara por extrapolación del uso de tecnologías por parte de las pymes. Según los resultados del índice global de competitividad (IGC), del Foro Económico Mundial (FEM), en 2010 Colombia se ubicó en el puesto 68 entre 139 países, lo cual representa un mejoramiento de la situación competitiva por segundo año consecutivo, al avanzar un puesto con respecto al resultado de 2009 y 7 puestos con respecto a 2008 (DNP, 2010a). Por otra parte, en lo referente a las tecnologías de la información y las comunicaciones, el informe del DNP dice:

\footnotetext{
En el pilar "preparación tecnológica” se registró una mejor posición competitiva gracias a una mejor percepción de los empresarios sobre la absorción tecnológica de las empresas, la mayor transferencia tecnológica derivada de la inversión extranjera directa y la disponibilidad de tecnologías de punta. Estos avances superaron el rezago en número de usuarios de Internet y los suscriptores de Internet de banda ancha. (DNP, 2010a, p. 4).
}

Ahora bien, antes de continuar debemos entender las tecnologías de la información y las comunicaciones (TIC), según la normatividad colombiana, “(...) como el conjunto de recursos, herramientas, equipos, programas informáticos, aplicaciones, redes y medios, que permiten la compilación, procesamiento, almacenamiento, 
transmisión de información, como voz, datos, texto, video e imágenes" (art. 6. , Ley 1341 de 2009).

En la actualidad hay un marcado optimismo en el desarrollo de las TIC y sus resultados, planteamiento eminentemente optimista, el cual se presenta en la nota introductoria del sitio del DANE que encabeza los informes estadísticos sobre las TIC en Colombia, la cual afirma:

El uso y la apropiación de las tecnologías de la información y la comunicación (TIC) por parte de las personas y los hogares inciden en las preferencias, la productividad y la dinámica interna de la unidad familiar conformada por personas que acceden y se apropian de estas tecnologías. Entre los impactos sociales más importantes del acceso y uso de las TIC en los hogares se encuentra la mayor oportunidad de acceso a información relevante para la satisfacción de necesidades básicas (oportunidades de trabajo y generación de ingresos), la conformación de redes de comunicación e información y la creación de capacidades. De aquí la importancia de monitorear la evolución del grado de adopción y uso de las TIC en Colombia. (DANE, 2010).

Sobre estas premisas, los diferentes gobiernos en las últimas décadas han venido adelantando iniciativas en materia de masificación de las TIC. En el país, las últimas mediciones arrojan los siguientes resultados: en conectividad a Internet, Colombia contaba con 6140271 de suscriptores para el cuarto trimestre de 2011, de los cuales, 4836833 tenían conexión a banda ancha (al cierre de 2014, según el portal Vive Digital, el país contaba con 9.8 millones de conexiones a Internet de banda ancha); en otras conexiones había 1303438 DE suscriptores. Según el MINTIC, la penetración total a nivel nacional de uso de la Internet era del 13.3 \%, y en banda ancha era del 6.6 \% (MINTIC, 2011). “Además, la incorporación de las TIC por particulares sigue siendo baja, por cuanto menos del $25 \%$ de la población tiene acceso a Internet en casa de acuerdo con el informe de conectividad del MINTIC” (DNP, 2012a).

Sin lugar a dudas, Colombia es un país sobre diagnosticado en todos los campos, y en el caso empresarial, no escapa a ello. Para empezar, "la esperanza de que los pueblos pasen del estado agrario saltándose la fase industrial tiene tantas posibilidades como las que un niño tiene de aprender a bailar sin antes andar" (Dertouzos, 1998). Aquí no se trata de que este sea un enfoque pesimista, sino más bien un 
enfoque realista, pues los informes sobre la situación de Colombia solo demuestran que el país está lejos de contar con una infraestructura en TIC aceptable y, por supuesto, esta situación se ve reflejada en las pymes (, CPC, 2012).

\section{La realidad de las cifras}

Los estudios y las encuestas realizados en el país demuestran que, efectivamente, no hay un avance significativo en la adopción de las tecnologías de la información y las comunicaciones (TIC) por parte de las pymes (, Mayorga, 2012; DANE, 2012; Vive Digital, 2012) y que los programas desarrollados por los diferentes gobiernos no han hecho mella en esta problemática. Las últimas encuestas así lo reafirman y los resultados de las mediciones internacionales también lo confirman (, CPC, 2012). Las causas son múltiples y van desde la corrupción imperante en el país hasta el total desconocimiento de los empresarios de las mipymes por las utilidades de las TIC.

Omar Perdomo, Cloud Sales Manager de Microsoft Colombia, considera que, "en algunos casos, la deficiencia en este asunto obedece a una cuestión de desconocimiento de los beneficios que, a través de nuevas tecnologías, pueden obtener las pymes y ser competitivas. En otros, es falta de asesoría, aunque también influye la poca inversión”. (Portafolio, 25 de mayo de 2012).

Y para reafirmar el anterior comentario desde los centros educativos:

Cada vez son más los directivos de las pequeñas y medianas empresas conscientes de la importancia de las herramientas tecnológicas, aunque muchas veces no saben cuáles son las más adecuadas para su tipo de negocio. Otros aún no tienen en cuenta que "la implementación de la tecnología abre múltiples posibilidades", dice Felipe Vega, de la consultoría Visión de la U. de La Sabana, especializada en pymes. (Naranjo, 22 de marzo de 2012).

El $45 \%$ de las pymes no están lista para TLC, es una de las conclusiones a la que llegó el último estudio del Politécnico Grancolombiano después de revisar los datos de 9200 microempresarios de todo el país. (El Espectador, 6 de mayo de 2012; Vega et al., 2011). 
El caso del desconocimiento de la realidad respecto a la adopción de las TIC por parte de las pymes y del desarrollo del país es latente y no está solo en cabeza de los pequeños empresarios, también en las agremiaciones que los representan, lo cual se nota cuando se encuentran expresiones como la del presidente de la Asociación Nacional de Empresarios de Colombia (ANDI), Bruce Mac Master, quien calificó a este Comité como el más futurista y aseguró que busca el fortalecimiento de las mipymes para generar más dinamismo económico y así incentivar el comercio electrónico; (a propósito del acuerdo entre el Ministerio de las TIC y la Andi, para trabajar de manera conjunta y permanente a través de un Comité 20/20 para acelerar el crecimiento del sector TIC en el país).(MINTIC, 2014). Luego está el informe del Foro Económico Mundial en el que se muestra que en 2011 el país ocupaba el lugar 58 entre las 138 naciones objeto de estudio en TIC. Y en otra declaración de los gremios más realista se afirma:

El expresidente de ACOPI, Juan Alfredo Pinto, estima que, si bien en materia de tecnología hay notorios avances y, en términos generales, se utilizan equipos y estructuras de comunicación y desarrollos informáticos, empero, la principal debilidad se presenta en la utilización de plataformas integradas y el manejo de herramientas de tecnologías de información, de manera que incluya todos los procesos de convergencia, plataformas como CRM, aplicaciones de organización de la producción o la logística de toda la cadena de abastecimiento. (Naranjo, 22 de marzo de 2012).

Y, por supuesto, el mismo Estado confirma el atraso en la adopción de las TIC por parte de las pymes. Solo el $15 \%$ de las pymes está conectado a Internet (, Mayorga, 2012; DANE, 2012). Y en declaraciones al diario El Espectador, el ministro Diego Molano dice: "Claro que las cifras muestran un panorama bastante desalentador: aunque más del $60 \%$ reconoce que la conexión a Internet es importante para consolidar su actividad" (E1 Mayorga, 2012).

En el portal Vive Digital (el sitio en Internet es gubernamental) consiste en "un plan de tecnología para los próximos cuatro años en Colombia (2014-2018), que busca que el país dé un gran salto tecnológico mediante la masificación de Internet y el desarrollo del ecosistema digital nacional" (Vive Digital, 2012). 
En el último informe sobre competitividad para 2012 del Consejo Privado de Competitividad, se destacan los diferentes índices internacionales de tecnologías de la información y las comunicaciones y la posición de Colombia en estas mediciones. De acuerdo con los últimos resultados del Anuario Mundial de Competitividad que elabora el Institute for Management Development (IMD), Colombia continuó su caída al pasar del puesto 46 en 2011 al puesto 52 en 2012, entre 59 países. Lo mismo ocurrió con los resultados del Indicador Global de Competitividad del World Economic Forum (WEF), que indican un retroceso del país de la posición 68 en 2011 a la posición 69 en 2012, entre 144 países, alejándose, por tanto, de la meta de ser uno de los países más competitivos de la región. (Concejo, 2012)

El índice de competitividad de la industria de TI, conocido como (EIU) por The Economist Intelligence Unit, básicamente compara varios países (66) de diferentes regiones del mundo en cuanto a la medida de las condiciones que ofrecen apoyo al desarrollo de la competitividad en las empresas de TI (The Economist Intelligence Unit, 2009). Para esto su medición se han desarrollado seis variables: entornos comerciales generales, infraestructura de TI, capital humano, investigación y desarrollo, entorno legal y apoyo público para el desarrollo industrial. En 2008 y 2009, Colombia estaba en el puesto 52 entre 66 países; en 2012, el informe colocó el país en el lugar 49; según el informe, algunas de las causales para que el país tenga esta posición son "el bajo desempeño en el entorno de investigación y desarrollo en TIC, así como también la aún limitada infraestructura que persiste en el país" (, CPC, 2012, p. 131).

El índice de desarrollo de TIC (IDI), generado por la Unión Internacional de Telecomunicaciones (UIT) - ya descrito en párrafos anteriores-, permite monitorear y comparar desarrollos en las TIC entre países, teniendo en cuenta la "preparación en infraestructura de los países para las TIC", el "uso intensivo de estas" y las "habilidades de los individuos, educación de la población mayor y porcentajes de matrículas en los diferentes niveles de formación”. Este último índice de los tres es el que tiene menor peso en la medición: entre 152 países, Colombia ocupó el puesto 76 en 2011.

En el caso de Colombia [...], al desagregar el pilar de preparación tecnológica, se observa una caída respecto al año anterior, indicando que para lograr el nivel de competitividad que requiere el país es indispensable fortalecer la promoción y 
uso de TIC en actividades diarias y en los procesos productivos, de manera que se traduzcan en mejoras en eficiencia y productividad. (, CPC, 2012, p. 132).

Finalmente, como se puede observar, las encuestas y los estudios establecen que, en definitiva, las mipymes cuentan con un rezago bastante significativo en la adopción de las tecnologías de la información y las comunicaciones y que hacen un uso muy superficial de estas, así lo confirman no solo el seguimiento de las entidades estatales y los estudios en general, sino también las conclusiones que se derivan de las tres más importantes mediciones internacionales de las TIC.

El Plan Nacional de Desarrollo 2010-2014 “Prosperidad para todos", del actual gobierno, centra su política en tres piedras angulares: más empleo, menos pobreza y más seguridad. El capítulo III trata sobre el "crecimiento sostenible"; en su literal A, "innovación para la prosperidad", contiene el numeral 1, "conocimiento e innovación”, el cual desarrolla cuatro lineamientos estratégicos basados en lo financiero, la formación y la organización. El cuarto está dedicado al "fomento y uso de las TIC", y en él se reconoce la importancia de estas, dado que son los instrumentos que apoyan la productividad y la competitividad del país. Y se destaca el hecho de que son estos instrumentos los que les permiten a los usuarios desempeñar un papel central en la innovación y promover la generación de conocimiento y nuevos negocios (DNP, 2012c).

El actual gobierno señala unas metas para incentivar la educación en torno a las TIC, dándole continuidad a los programas del MINTIC, ampliando los esfuerzos de capacitación del Sena y mejorando su calidad en la formación, estimulando el uso de la infraestructura y los servicios de las TIC con el fin de aumentar la cobertura, generando beneficios para las empresas que inviertan en investigación y desarrollo (ya implementado mediante el art. 158-1 del Estatuto Tributario Nacional, recientemente modificado por la Ley 1450 de 2011). Pero, lamentablemente, las metas son muy modestas. En el mismo documento se señalan las aspiraciones para beneficiar con el incentivo tributario antes descrito a 300 pymes, o los beneficiarios de formación doctoral en 3000. En el evento Mipymes Digital 2012, celebrado en Bogotá, la aspiración era mostrar a aproximadamente 3000 microempresarios lo último en tecnología para su desarrollo; sobre una cifra de 1146420 microempresas, cualquier número que se presente en cuanto a la capacitación, aumento de 
conexiones a Internet o recursos para tecnología es grande y llamativa, pero mientras no sea la totalidad o buena parte de esta, no pasará de ser más que demagogia.

Mientras tanto, los gobiernos siguen haciendo esfuerzos por asumir las TIC en todos los niveles. Como se indicó en uno de los párrafos anteriores, una de las características más relevantes del uso de la Internet es el acceso a la información, y para los microempresarios dependientes de otro tipo de necesidades y afanes esta no cuenta.

$\mathrm{El}$ avance desaforado de las TIC nos pone es la difícil coyuntura entre decidir por suplir las necesidades básicas de una mayoría de la población sacrificando las inversiones en tecnología y, por supuesto, quedando cada vez más rezagados, o el desarrollo de instrumentos tecnológicos para el disfrute de una minoría. Encontrar el balance adecuado es la tarea de los gobiernos de los países en vías de desarrollo.

\section{Conclusiones}

Todas las encuestas y los estudios dejan ver de lejos que Colombia cuenta con un rezago bastante significativo en cuanto a la adopción de las tecnologías de la información y las comunicaciones, el cual está confirmado no solo por el seguimiento de las entidades estatales, sino también por las tres más importantes mediciones internacionales de las TIC.

El único rubro que se encuentra con una gran penetración es la telefonía móvil, pero, al mismo tiempo, los servicios son costosos y deficientes y no hay una amplia cobertura a nivel nacional; por otra parte, falta más desarrollo de contenidos y utilidades de la telefonía móvil.

El país también carece de una frágil infraestructura y potencial para desarrollar las TIC y para su adopción.

Por consiguiente, el rezago que presenta el país en las TIC se ve reflejado en las pymes en todos los nivele, y, en forma reiterada, confirmado por las encuestas y los estudios.

Según las encuestas, el uso que se les da a las TIC es superficial y hay desaprovechamiento y desconocimiento de las capacidades de los sistemas computacionales y de los diferentes productos para usos empresariales.

Si bien es cierto que ha habido algunos avances, estos no son suficientes para que el país salga adelante; se necesita una gran inversión en capacitación masiva en diferentes temas empresariales, pero, ante todo, en el uso de las TIC. 
Hace falta un seguimiento más puntual sobre el uso y aprovechamiento de los sistemas computacionales y de programas por parte de las pymes, lo cual puede llevar a un mejor diagnóstico al respecto.

Finalmente, la India es un ejemplo del grado y el tamaño de las iniciativas para formar un gran potencial en TIC, tanto en lo humano como en lo técnico y tecnológico; pero esto no quiere decir ni asegura que el país salga adelante y logre alcanzar los niveles de vida de su población como los de los países desarrollados, a menos que se tome realmente en serio el crecimiento sostenido y constante, muy por encima de la media mundial (Dertouzos, 1998). Por lo tanto, centrar el progreso de las naciones alrededor de las TIC, olvidando o dejando de lado sus ventajas competitivas, nos llevará por caminos inciertos. Por ende, debe armonizarse el desarrollo de las TIC en paralelo al servicio del potencial económico de un país.

En los propósitos del Plan Nacional de Desarrollo 2010-2014 se le da una gran importancia a la competitividad y al papel que desempeñan las pymes. El gobierno pretende, para los próximos años, alcanzar y sostener un crecimiento de más del $6 \%$ para lograr un verdadero desarrollo y centrar sus expectativas en la competitividad de las pymes; pero, finalmente, esta es una debilidad enquistada en las pymes, que se convierte en una de las principales falencias que les impide ser más productivas y más competitivas.

\section{Referencias}

Acosta, J. (2012). El necesario reconocimiento del principio de justicia contractual. Revista Virtual Via Inveniendi et Iudicandi, 7(1)-54. Recuperado de goo.g1/XcqbhG

CCIT y Fedesarrollo. (2013). El papel de las TIC en el desarrollo de la pequeña empresa: reflexiones de política a la luz del caso colombiano. Coyuntura TIC (diciembre). Recuperado de goo.g1/jLg2xH

Congreso de la República de Colombia. (2 de agosto de 2004). Ley 905 de 2004. Por medio de la cual se modifica la Ley 590 de 2000 sobre promoción de desarrollo de la micro, pequeña y mediana empresa colombiana y se dictan otras disposiciones. Diario Oficial $n .{ }^{\circ} 45.628$ de 2 de agosto de 2004 Recuperado de goo.gl/xAAess 
. (30 de julio de 2009). Ley 1341 de 2009. Por la cual se definen principios y conceptos sobre la sociedad de la información y la organización de las Tecnologías de la Información y las Comunicaciones -TIC-, se crea la Agencia Nacional de Espectro y se dictan otras disposiciones. Diario Oficial n. 47426 de julio 30 de 2009. Recuperado de goo.gl/sV5S3z

. (16 de junio de 2011). Ley 1450 de 2011. Por la cual se expide el Plan Nacional de Desarrollo, 2010-2014. Diario Oficial n. 48.102 de 16 de junio de 2011. Recuperado de goo.gl/RkHFiQ

Consejo Superior de Pequeña y Mediana Empresa. (19 de abril de 2013). Acuerdo número 02 de 2013. Por el cual se modifica el artículo cuarto del Acuerdo n. 01 del Consejo Superior de Pequeña y Mediana Empresa. Recuperado de goo.g1/CWEVd3

Departamento Administrativo Nacional de Estadística (DANE). (junio 7 de 2010). Indicadores básicos de tecnologías de la información y la comunicación. Año 2010. Tenencia y uso de TIC en hogares y por personas de 5 años y más (Boletín de prensa). Recuperado de goo.gl/sNlrzG

. (2011). Tecnologías de la Información y las Comunicaciones -TIC-. Recuperado de goo.gl/ermlDM

Departamento Nacional de Planeación (DNP). (2010a). Reporte Global de Competitividad del Foro Económico Mundial 2010-2011. Resultados para Colombia - 2010. Recuperado de goo.gl/7CJPyQ

. (2010b). Plan Nacional de Desarrollo 2010-214 "Prosperidad para todos" (Tomos Iy II). Bogotá: DNP. Recuperado de goo.gl/rlJVSr

. (2012a). Reporte Global de Tecnología de la Información 2012. Foro Eco-

nómico Mundial. Recuperado de http://www3.weforum.org/docs/GITR/2012/ WEF_NR_GITR_2012_LatinAmerica_SP.pdf

file://Users/jrpuentesg/Downloads/informe\%20nacional\%20de\%20competividad-2012-2013\%20-\%20consejo\%20privado\%20de\%20competitividad.pdf (Concejo, 2012)

. (2012b). Estadísticas y estudios micro, pequeñas y medianas empresas. Censo económico 1990 y 2005. Recuperado de goo.gl/TPEYfA

(2012c). Plan Nacional de Desarrollo 2010-2014. "Prosperidad para todos”. Bogotá: DNP. Recuperado de goo.g1/K3dbcI

Dertouzos, M. L. (1998). Qué será. Bogotá: Planeta.

E1 Espectador. (6 de mayo de 2012). El 45 \% de las pymes no están listas para el TLC. El Espectador-Redacción Negocios. Recuperado de goo.g1/5hLVo2 
Flores, G. (2014). La validez jurídica de los documentos electrónicos en Colombia a partir de sus evolución legislativa y jurisprudencial. Revista Verba Iuris, 31, pp. 43-71. Recuperado de goo.gl/sT8yhK

Foro Económico Mundial. (2009). Reporte Global de Tecnologias de la Información.

Harvard University, Technologies Group y Center for International Development. (2000). Preparación para el mundo interconectado. Una guia para los países en desarrollo. Recuperado de goo.g1/JkTS9J

Mayorga, D. (24 de mayo de 2012). Solo el 15 \% de las pymes están conectadas a Internet. El Espectador Recuperado de goo.gl/JErtXC

Ministerio de Comercio, Industria y Turismo. (2008). Resolución 3205 de 2008. Por medio de la cual se reglamentan los Consejos Regionales.

. (15 de enero de 2013). Acuerdo número 01 de 2013. Por el cual el Consejo Superior de Pequeña y Mediana Empresa asume las funciones del Comité Nacional Interinstitucional OBAPYME. Recuperado de goo.g1/KzlnKv

Ministerio de Tecnologías de la Información y las Comunicaciones (MINTIC). (2011). Estadísticas del sector. Sector TIC. Recuperado de goo.g1/W5dCdI /

http://www.mintic.gov.co/portal/604/w3-article-5958.html (MINTIC). (2014)

Moreno, A. J. (Ed.). (2012). Informe Nacional de Competitividad 2011-2012. Consejo Privado de Competitividad Colombia. Bogotá: Nomos S. A.

Naranjo, F. (22 de marzo de 2012). Pymes deben invertir en tecnología para poder competir. Portafolio. Recuperado de goo.gl/SFmfiS

Negroponte, N. (1995). Ser digital. Buenos Aires: Atlántida.

Palomares, J. (2012). Normas de comercio electrónico en materia de seguridad y privacidad y su reflejo en Colombia. Revista Virtual Via Inveniendi et Iudicandi, 7(1), Recuperado de goo.g1/4ovFHX

Pardo, D. (11 de junio de 2012). Por qué Internet es tan costosa en América Latina. BBC Mundo. Recuperado de goo.g1/K3wR28

Portafolio. (24 de mayo de 2012). Gobierno anuncia $\$ 32.000$ millones en tecnología para pymes. Portafolio. Recuperado de goo.gl/9QrcZR

Portafolio. (25 de mayo de 2012). Uso de las TIC, un salto a la modernidad. Portafolio. Recuperado de http://www.portafolio.co/negocios/uso-las-tic-un-salto-la-modernidad Puentes, J. R. (2013). Las pymes colombianas y las TIC $\dot{\dot{\zeta}}$ Panacea o Ilusión? Bogotá: Ed. Temis.

Puyana, D. G. (2012). La problemática de las pymes en Colombia: internacionalizarse o morir. Recuperado de goo.g1/EUnBtJ 
Secretaría General de la Comunidad Andina. (julio de 2012). Resolución 1487 de 2012. Reglamento de Funcionamiento del Observatorio Andino de la MIPYME (OBAPYME). Gaceta Oficial, año XXIX, n. ${ }^{\circ}$ 2075. Recuperada de goo.g1/BQIPyQ

The Economist Intelligence Unit. (2009). Resistencia en medio de la confusión. Benchmarking la competitividad en la industria del TI 2009. Londres, Nueva York, Hong Kong: The Economist Intelligence Unit y BSA. Recuperado de goo.g1/PN748v

Universidad de los Andes. (25 de febrero de 2009). Metodología de monitoreo de Gobierno en Línea en Colombia. Bogotá: Universidad de los Andes y Ministerio de Comunicaciones. Recuperado de goo.gl/kWVAbJ

Velásquez, D. y Moreno, A. (2015). Aspectos de la regulación TIC en Colombia. Revista IUSTA, 2(43), 139-157. Recuperado de goo.gl/Q3Wvq8

Vega R., R. A.; Castaño R., A. Y Mora R., R. J.: Pymes . Reflexiones para la pequeña y mediana empresa en Colombia, Bogotá. Politécnico Grancolombiano, 2011.

Vive Digital. (2012). Colombia en el contexto internacional. Recuperado de http://www.mintic.gov.co/portal/vivedigital/612/w3-propertyvalue-19437.html Welles. O. (1949). 1984. Reino Unido: Harvill Secker. 